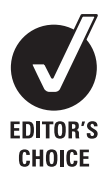

See Editorial, p 2

${ }^{1}$ Health Protection Agency Centre for Infections, London, UK: ${ }^{2}$ Division of Population Health, University College London, London, UK;

${ }^{3}$ Department of Virology, University College London, London, UK

Correspondence to: Ms A E Brown, HIV/STI Department, Health Protection Agency Centre for Infections, 61 Colindale Avenue, London NW9 5E0, UK; Alison.brown@hpa. org.uk

Accepted 13 October 2008 Published Online First 27 October 2008

\title{
Implications for HIV testing policy derived from combining data on voluntary confidential testing with viral sequences and serological analyses
}

\author{
A E Brown, ${ }^{1,2}$ G Murphy, ${ }^{1}$ G Rinck, ${ }^{1}$ J P Clewley, ${ }^{1}$ C Hill, ${ }^{1}$ J V Parry, ${ }^{1}$ A M Johnson, ${ }^{2}$ \\ D Pillay, ${ }^{1,3} 0 \mathrm{~N} \mathrm{Gill}^{1}$
}

\begin{abstract}
Objectives: Laboratory, clinical and sequence-based data were combined to assess the differential uptake of voluntary confidential HIV testing (VCT) according to risk and explore the occurrence of HIV transmission from individuals with recently acquired HIV infection, before the diagnostic opportunity.
\end{abstract}

Methods: Between 1999 and 2002, nearly 30000 anonymous tests for previously undiagnosed HIV infection were conducted among men who have sex with men (MSM) attending 15 sentinel sexually transmitted infection (STI) clinics in England, Wales and Northern Ireland. Using a serological testing algorithm, undiagnosed HIV-infected men were categorised into those with recent and non-recent infection. VCT uptake was compared between HIV-negative, recently HIV-infected and nonrecently HIV-infected men. A phylogenetic analysis of HIV pol sequences from 127 recently HIV-infected MSM was conducted to identify instances in which transmission may have occurred before the diagnostic opportunity.

Results: HIV-negative MSM were more likely to receive VCT at clinic visits compared with undiagnosed HIVinfected MSM (56\% (14 020/24 938) vs 31\% (335/ 1072); $p<0.001)$. Recently HIV-infected MSM were more likely to receive VCT compared with those with nonrecent infections (42\% (97/229) vs $28 \%$ (238/844); $\mathrm{p}<0.001) .22 \%$ (95/425) of undiagnosed HIV-infected MSM with STI received VCT. Phylogenetic analysis revealed at least seven transmissions may have been generated by recently HIV-infected MSM: a group that attended STI clinics soon after seroconversion.

Conclusions: The integration of clinical, laboratory and sequence-based data reveals the need for specific targeting of the recently HIV exposed, and those with STI, for VCT. VCT promotion alone may be limited in its ability to prevent HIV transmission.

Sequence-based, laboratory and clinical data are collected routinely around HIV diagnoses for medical and surveillance purposes. In particular, sequence-based antiretroviral resistance testing has recently become routine to inform treatment options. ${ }^{1}$ This has resulted in an accumulation of HIV pol sequences, the variability of which allows phylogenetic reconstruction of possible transmission events. ${ }^{2}$ Clinical data (eg, co-infection with sexually transmitted infections (STI) etc) allows the ascertainment of risk factors associated with infection. Laboratory methods such as the serological testing algorithm for recent HIV seroconversion (STARHS) ${ }^{34}$ can identify men who have sex with men (MSM) with recent HIV infection. Such men are likely to have heightened infectivity as a result of the elevated viral load shortly after infection. ${ }^{5}{ }^{6}$ Combining such data has the potential to enhance our understanding of HIV transmission and its associated risk factors to a greater extent than can be gleaned from each data source when considered individually

Methods have combined laboratory and clinical data previously. ${ }^{7-9}$ Pao et al used phylogenetic analysis of HIV pol sequences to identify possible transmissions between MSM and, through linkage to clinical data, identified risk factors associated with transmission. Brenner et al $l^{9}$ recently linked sequences to infection stage data in a phylogenetic analysis that highlighted the higher transmission potential from individuals who were recently HIV infected. Such studies remain exploratory, but demonstrate the benefits of combining data sources to enhance our understanding of HIV transmission.

Combined approaches could also be focused to examine specific public health problems. For example, assessing the effectiveness of voluntary confidential HIV testing (VCT) in preventing HIV transmission, which we explore in this paper. VCT promotion among MSM who may have had HIV exposure is an important part of the UK response to the HIV epidemic and remains a central component of best practice guidelines ${ }^{10}$ and the sexual health strategy. ${ }^{11}$ Prompt HIV diagnosis may prevent further HIV transmission through ensuring patients' viral load is low (through regular monitoring and/or administering antiretroviral therapy when clinically appropriate ${ }^{12}$ ) and providing earlier opportunities for partner notification and behaviour change counselling. Between 1996 and 2006 the proportion of MSM attendees of STI clinics receiving VCT rose from $45 \%$ to $85 \%{ }^{13}$ However, during the same period, annual HIV incidence has remained at approximately $3 \% .{ }^{13}$ Therefore, the effectiveness of promoting VCT to prevent HIV transmission may be limited for reasons that are not fully understood.

We examine the benefits of integrating sequence and laboratory data derived from an unlinked anonymous (UA) survey of STI clinic attendees ${ }^{14}$ to improve our understanding of the effectiveness of VCT in preventing HIV transmission. VCT uptake is compared between recently and non-recently HIV-infected MSM to assess differential uptake according to transmission risk. A phylogenetic analysis is conducted to explore the occurrence of HIV transmission from recently HIV-infected individuals before the diagnostic opportunity. 


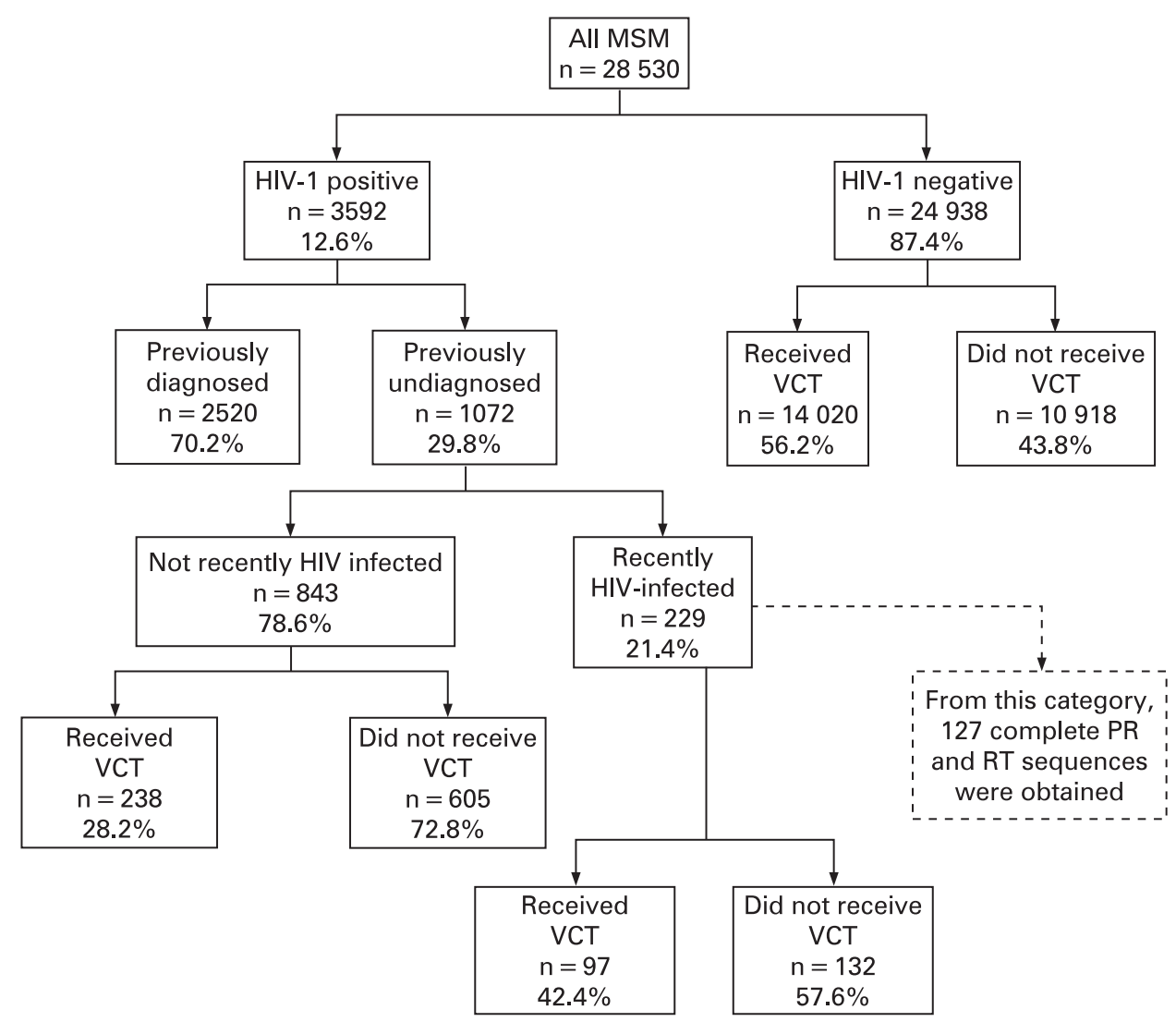

Figure 1 Flow diagram of all men who have sex with men (MSM) attending 15 sexually transmitted infection clinics: England, Wales and Northern Ireland, 1999-2002. PR, protease; RT, reverse transcriptase; VCT, voluntary confidential HIV testing.

\section{METHODS}

\section{Study population}

Data and sera were obtained from the UA survey between 1999 and 2002. ${ }^{14}$ Established in 1989, this ongoing survey measures HIV prevalence (including undiagnosed prevalence) among attendees of sentinel STI clinics (15/232 clinics in England, Wales and Northern Ireland; seven in London, eight elsewhere). Such clinics provide advice, treatment and screening services for HIV/STI. Blood left over from syphilis testing is irreversibly unlinked from patient identifiers and anonymously HIV tested. Patients can only be included in the survey once per calendar quarter. Limited information is retained with each sample: risk group; age group; sex; calendar quarter of attendance; clinic; birth region; STI diagnosis; whether patient was HIV diagnosed before the clinic attendance or had received VCT during the clinic attendance. ${ }^{15}$ The UA survey has had Public Health Laboratory Service ethical approval since its inception in 1989 and is compliant with the Human Tissue Act (2004).

\section{Diagnosis status and HIV testing uptake}

Patients found to be HIV positive through UA testing were categorised as "newly diagnosed" or "undiagnosed" according to whether they received VCT at the attendance (patients diagnosed with HIV before the clinic attendance were excluded). The "newly diagnosed" received VCT during the clinic attendance and the "undiagnosed" did not receive VCT, leaving the clinic remaining undiagnosed. T tests were used to supplement results when appropriate.
Serological testing algorithm for recent HIV seroconversion All samples from MSM with a newly diagnosed or undiagnosed HIV infection were tested using STARHS. ${ }^{3}{ }^{4}$ STARHS distinguishes recent HIV infections through exploiting evolving antibody responses. The algorithm assumes anti-HIV titres increase at a similar rate between individuals over the months following infection: a less sensitive anti-HIV-1 enzyme immunoassay (EIA) is applied to test serum or plasma specimens confirmed to be anti-HIV-1 positive using a sensitive EIA. Reactivity below the cut-off (standardised optical density 1.0) in the less sensitive EIA indicates that the sample was derived from someone who was infected an average of 170 days (95\% CI 162 to 183 days) before specimen collection. ${ }^{16}$

\section{Phylogenetic analysis}

Samples from recently HIV-infected individuals were genotyped as previously described. ${ }^{17}$ The protease region of pol was sequenced along with the first 230 codons of reverse transcriptase (RT). Protease and RT sequences were aligned using sequence alignment version 2 across 998 nucleotides. Primary drug resistance mutation sites ${ }^{18}$ were removed from the alignment to eliminate bias from convergent evolution. Phylogenetic analysis was performed using phylogenetic analysis using parsimony ${ }^{19}$ with a neighbour-joining algorithm. The alignment was run through ModelTest to select the best fitting evolutionary model. A heuristic search was conducted for a maximum likelihood tree using the selected model $(\mathrm{GTR}+\mathrm{I}+\mathrm{G})$ and its derived parameters (proportion of invariable sites 0.43 and gamma distribution 0.79) using the neighbour-joining tree as the starting tree. A bootstrap analysis 


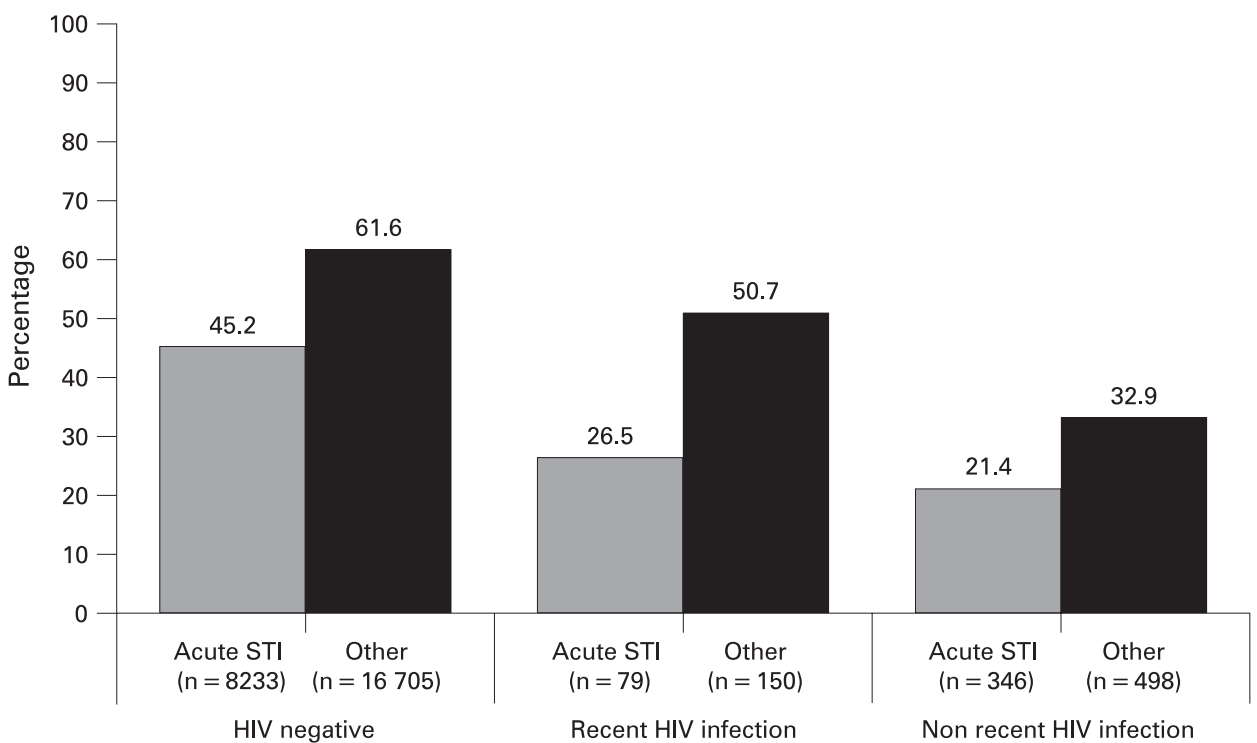

Figure 2 Proportion of men who have sex with men attending 15 sexually transmitted infection (STI) clinics receiving voluntary confidential HIV testing, by diagnosis status: England, Wales and Northern Ireland, 1999-2002.

(500 replicates) was used to obtain statistical support for branching patterns. Genetic distances were calculated from the consensus tree for each terminal cluster. Branches comprising two or more sequences with an average bootstrap value of over $99 \%$ and an average genetic distance under 0.015 nucleotide substitutions per site were considered to be "possible transmissions". 8

\section{RESULTS}

\section{Population characteristics}

Between 1999 and 2002, 28530 UA tests were conducted on samples from MSM attending 15 STI clinics in England, Wales and Northern Ireland (fig 1). Of these, 3592 samples were found to be HIV positive, of which 1072 were derived from patients with a previously undiagnosed infection (newly diagnosed or undiagnosed HIV infection). Among this group, 21\% (229) of samples were derived from recently infected MSM. Complete protease and RT sequences were obtained from 127 samples from recently HIV-infected patients (the remaining 102 could not be amplified because of problems associated with using residual samples).

Overall, of the 229 samples from recently HIV-infected MSM, 86\% (196) attended clinics in London, 56\% (127) were UK born and $15 \%$ (35) were born elsewhere in Europe. For the 127 linkable to sequences, equivalent figures were $86 \%$ (109), 58\% (74) and $15 \%(19)$, respectively.

\section{VCT uptake}

VCT uptake was higher among MSM from whom HIV-negative samples were collected than compared with samples from MSM with previously undiagnosed HIV infection: 56\% (14 020/ 24 938, 95\% CI 55.6 to 56.8) versus 31\% (335/1072, 95\% CI 28.5 to $34.1 ; p<0.001$ ) (fig 1$)$. Forty-two per cent $(97 / 229,95 \%$ CI 36.1 to $48.8 \%$ ) of MSM with samples indicating recent HIV infection received VCT. This compares with 28\% (238/843, 95\% CI 25.3 to $31.3 ; p<0.001)$ among samples from previously undiagnosed HIV-infected MSM with a non-recent infection.

Figure 2 demonstrates the differential VCT uptake according to transmission risk. Regardless of HIV infection, all MSM were less likely to receive VCT if they had an STI: uptake was $22 \%$
(95/425, 95\% CI 18.7 to 26.6$)$ among the previously undiagnosed HIV infected and 45\% (3721/8233, 95\% CI 44.1 to 46.3$)$ among the HIV negative $(p<0.001)$.

\section{Phylogenetic analysis}

Of the 127 sequences from samples from recently HIV-infected MSM, 16 clustered with at least one other sequence with a bootstrap support of at least $99 \%$ and a genetic distance under 0.015 nucleotide substitutions per site (fig 3). These formed seven clusters $(A-G)$ : cluster A contained four sequences and clusters B-G each consisted of sequence pairs. Cluster B consisted of sequences obtained in Wales and the remaining six were from London. Clusters $\mathrm{A}-\mathrm{D}$ and $\mathrm{F}$ contained sequences from samples obtained within the same, or successive, calendar quarters.

Clusters C, E and G were composed entirely of sequences from individuals whose HIV infection was diagnosed at that STI clinic attendance (fig 3). Clusters B and D comprised sequences from individuals who left the clinic remaining unaware of their HIV infection. Eight sequences that fell into a cluster were derived from patients who had an STI.

\section{DISCUSSION}

More than half of recently HIV-infected MSM and nearly $80 \%$ of all undiagnosed HIV-infected MSM with an STI, attending clinics collaborating in the UA survey, did not receive VCT. Phylogenetic analyses of HIV pol sequences from recently HIVinfected MSM identified at least seven possible instances of HIV transmission before the diagnostic opportunity.

\section{VCT uptake}

Combining VCT data with laboratory algorithms demonstrated that using VCT uptake as a prevention indicator lacks sensitivity in discriminating between groups with varied transmission risks. The diagnostic opportunity was missed among the recently HIV infected and those with an STI. We speculate that this could be for several reasons. First, recently HIV-infected individuals may have previously tested HIV negative and consequently believed themselves still to be HIV negative. Second, a concern that testing within a 3-month 

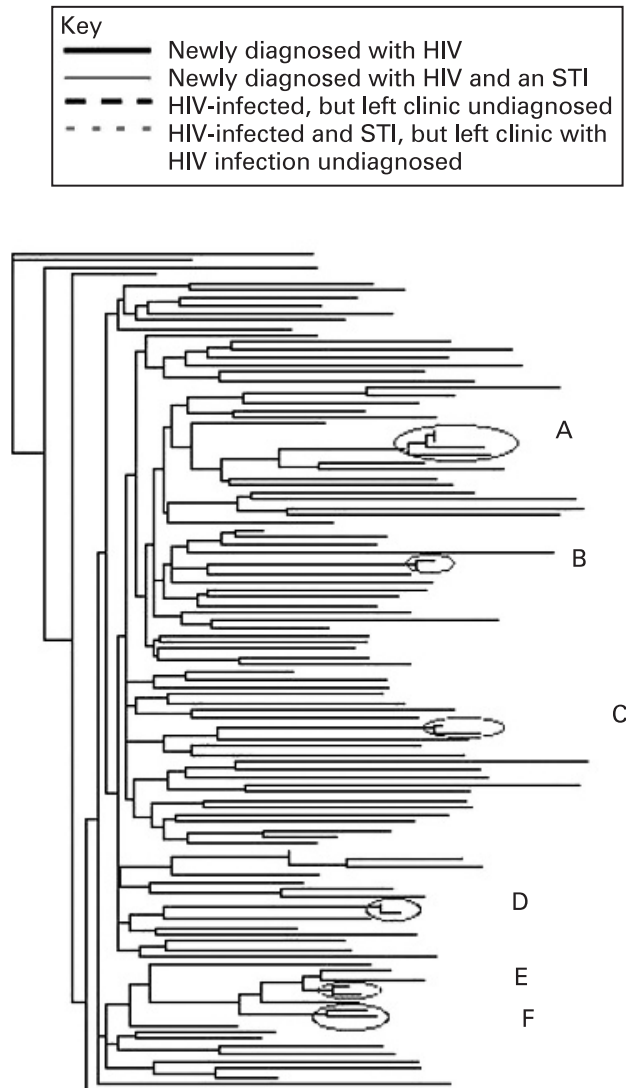

1 Not to scale

2 Within phylogenetic clusters, the difference between the quarter of attendances between patients from

whom the sequences were obtained. The earliest quarter if presented as " 0 ", and the next

chronologically, by quarter count. For example, a phylogenetic cluster of three sequences from patients

with quarters of attendance of "2001 Q2, 2001 Q4 and 2003 Q3" would be presented "0, 2, 9"

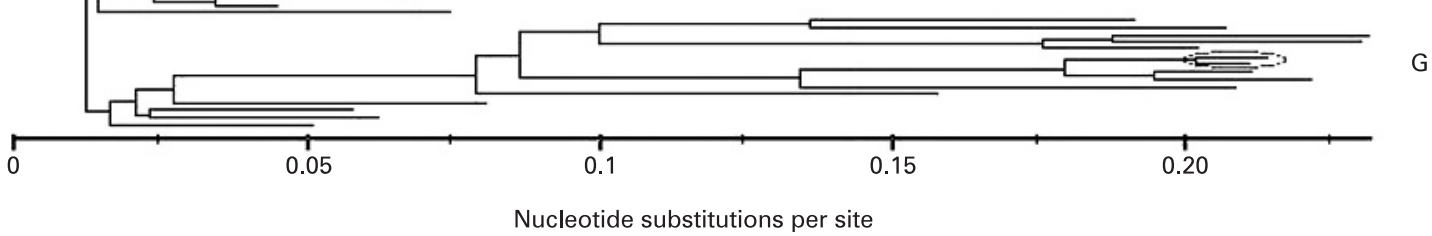

Figure 3 Possible transmission events among recently HIV-infected patients attending 15 sexually transmitted infection (STI) clinics: England, Wales and Northern Ireland, 1999-2002.

window period would affect diagnostic accuracy ${ }^{20}$ may have led to test deferral among the recently HIV infected. Third, testing algorithms may be failing to pick up those at risk. Fourth, a clinical focus on STI management may have detracted from those with an STI receiving VCT. Finally, there may exist a subset of MSM who are serial VCT refusers.

It is only known whether VCT was received; it is not known whether and why VCT was not offered, or was refused. No data were collected concerning past HIV testing history. Furthermore, only patients undergoing syphilis tests are included: a population not necessarily representative of the clinic population. The survey design may mean a small subset of patients are included many times within the dataset. The size and attributes of this group are not known, but if they are associated with a particular risk or HIV testing behaviours, this may affect data accuracy.

Data are taken from 1999-2002, a period in which VCT uptake was lower among MSM. A British Association for Sexual Health and HIV (BASHH) audit demonstrated a recent rise in the proportion of STI clinic attendees being HIV tested (as a result of better targeted HIV testing efforts (including patients with STI) and more assertive testing invitations (eg, opt-out policies).$^{20}$ Although this increased VCT uptake is reflected within the UA survey, which found that VCT uptake increased to $85 \%{ }^{13}$ overall in 2006 , in the same year VCT was measured at $42 \%$ among HIV-infected MSM with an STI and 63\% among the recently HIV infected. Therefore, despite an increased VCT uptake generally, a substantial proportion of those at highest risk of onward transmission still leave the clinic remaining undiagnosed.

\section{Transmission before diagnostic opportunity}

The phylogenetic analysis was conducted to observe instances in which transmission events may have occurred before the diagnostic opportunity. No attempts were made to calculate the force of transmission from the recently HIV-infected population because the size and inconsistent geographical make-up of the dataset prohibits this, and indeed any extrapolation of results to the HIV-infected population.

We observed seven distinct possible HIV transmission clusters between recently HIV-infected MSM. Five clusters had closeness in the likely seroconversion dates between the sequences involved. This demonstrates the potential for HIV transmission to occur rapidly from recently HIV-infected MSM, even among those who attend clinics soon after infection. Combining 


\section{Key messages}

- Between 1999 and 2002, VCT uptake was higher among HIVnegative MSM, compared with HIV-infected MSM. Low VCT uptake was observed among recently HIV-infected MSM.

- Phylogenetic reconstruction of HIV transmission events among recently HIV-infected MSM indicate that transmissions may have occurred before the opportunity for VCT.

- VCT promotion alone may be limited in its ability to prevent HIV transmission.

- Integrating clinical, laboratory and sequence-based HIV data can enhance our understanding of HIV transmission.

sequences with VCT data demonstrated that approximately half of the MSM involved in a possible transmission went on to miss the diagnostic opportunity at their clinic attendance, increasing the risk of further transmission and limiting the opportunity for prompt partner notification.

It cannot be conclusively demonstrated that the transmission clusters identified represent actual transmissions events. The bootstrapping and genetic distance cut-offs used are not definitive but have been shown to reduce the proportion of false-positive clusters among phylogenetic analyses of UK HIV sequences. ${ }^{21}$ The analysis cannot rule out that each cluster could have been generated by a third party (not sampled) infecting each sequence in the cluster, or acting as an intermediary between the sequences. Similarly, transmissions from individuals in the dataset may not be represented if the infection generated was not in the study.

The survey methodology allows patients to be sampled (within the same clinic) up to four times annually, but not within the same calendar quarter. Therefore, theoretically, the transmission clusters identified could have been formed between sequences from the same individual, sampled many times. Furthermore, the UA survey is necessarily designed to prevent the identification of individuals. However, five of the possible transmissions $(C-G)$ involve patient pairs who have a different birth region or age group (fig 3), making it unlikely that the sequences come from the same person. Similarly, there must be at least two individuals included in cluster A-but not necessarily four. The patients in cluster B both attended the same clinic within the same calendar quarter, meaning they should be different patients.

\section{Implications for HIV testing policy}

BASHH guidelines (2006) recommend that all patients should be offered VCT on their first clinical attendance, ${ }^{10}$ including patients presenting with STI. Whereas the benefits of VCT are unquestionable for clinical purposes, the public health benefits require further consideration. Since 2002, HIV testing policy has not been adapted specifically to target patients with recent risk exposure. ${ }^{10}$ BASHH guidelines recommend that recently HIVexposed patients receive two HIV tests: one immediately and one 3 months after exposure (the " 3 -month rule"). This is because of concerns that HIV testing shortly after exposure may not yield accurate results. However, recent improvements in HIV test sensitivity mean that the latest (so-called "fourth generation" tests) can detect both anti-HIV and HIV p24 antigen within 4 weeks of infection. ${ }^{22}$ We believe that delaying the second test for 3 months may be detrimental. The BASHH audit found the most commonly cited reason for not receiving VCT was deferral because of concerns relating to the accuracy of HIV testing shortly after exposure: ${ }^{20}$ the rate of reattendance for the second test was low. We suggest the guidelines should be modified to emphasise the importance of undertaking VCT at first attendance. If the first test is negative, the second test should be brought forward to 1 month post-attendance (provided fourth generation tests are employed) to enable earlier interventions to reduce onward transmission.

Our phylogenetic analysis illustrates the potential for the recently HIV infected to generate rapid onward transmission, even among those who receive VCT soon after infection. Consequently, VCT alone may not have a large impact on the greater transmission potential of recently HIV-infected MSM. Alternative strategies include: more rigorous partner notification; post-exposure prophylaxis among recently exposed MSM; encouraging frequent, regular testing; educating MSM and healthcare providers about seroconversion illness.

This analysis demonstrates how the integration of clinical, laboratory and sequence-based data can be used to explore HIV transmission and related public health problems more extensively. Although the analysis has not measured the force of infection from the recently HIV-infected population, or the impact of VCT on HIV transmission, it highlighted the need for specific targeting of the recently exposed and those with STI for VCT. It also revealed that VCT alone may be limited in its ability to prevent HIV transmission.

Acknowledgements: The authors extend many thanks to their colleagues at the 15 participating STI clinics and associated laboratories, without whom this work would not have been possible.

Funding: This study was funded by the Department of Health, award ref UAS 6/1, for which the authors are very grateful.

\section{Competing interests: None.}

Ethics approval: The UA survey has had Public Health Laboratory Service ethical approval since its inception in 1989 and is compliant with the Human Tissue Act (2004).

The manuscript was submitted before the publication of updated BASHH HIV testing guidelines in September 2008. These guidelines can be found at: http://www.bashh.org/ documents/1838.

\section{REFERENCES}

1. Pozniak A, Gazzard B, Anderson J, et al. British HIV Association (BHIVA) guidelines for the treatment of HIV-infected adults with antiretroviral therapy. HIV Med 2003;4(Suppl 1):1-41.

2. Hue S, Clewley JP, Cane PA, et al. HIV-1 pol gene variation is sufficient for reconstruction of transmissions in the era of antiretroviral therapy. AIDS 2004;18:719-28.

3. Murphy G, Charlett A, Jordan JF, et al. HIV incidence appears constant in men who have sex with men despite widespread use of effective antiretroviral therapy. AIDS 2004;18:265-72.

4. Janssen RS, Satten GA, Stramer SL, et al. New testing strategy to detect early HIV1 infection for use in incidence estimates and for clinical and prevention purposes. JAMA 1998;280:42-8.

5. Wawer MJ, Gray RH, Sewankambo NK, et al. Rates of HIV-1 transmission per coital act, by stage of HIV-1 infection, in Rakai, Uganda. J Infect Dis 2005;191:1403-9.

6. Gray RH, Wawer MJ, Brookmeyer R, et al. Probability of HIV-1 transmission per coital act in monogamous, heterosexual, HIV-1-discordant couples in Rakai, Uganda. Lancet 2001;357:1149-53.

7. Pao D, Fisher M, Hue S, et al. Transmission of HIV-1 during primary infection: relationship to sexual risk and sexually transmitted infections. AIDS 2005;19:85-90.

8. Hue S, Pillay D, Clewley JP, et al. Genetic analysis reveals the complex structure of HIV-1 transmission within defined risk groups. Proc Natl Acad Sci U S A 2005;102;4425-9.

9. Brenner BG, Roger M, Routy JP, et al. High rates of forward transmission events after acute/early HIV-1 infection. J Infect Dis 2007;195:951-9.

10. British Association for Sexual Health and HIV British Infection Society. UK National Guidelines for HIV Testing. British HIV Association, 2006.

11. Department of Health. The national strategy for sexual health and HIV, 2001. London. http://www.dh.gov.uk/en/Publicationsandstatistics/Publications/ PublicationsPolicyAndGuidance/DH_4003133?IdcService=GET_FILEGdID= 5539\&Rendition=Web/ (accessed Dec 2008). 
12. Ledergerber $\mathbf{B}$, Egger $\mathrm{M}$, Opravil $\mathrm{M}$, et al. Clinical progression and virological failure on highly active antiretroviral therapy in HIV-1 patients: a prospective cohort study. Swiss HIV Cohort Study. Lancet 1999;353:863-8.

13. The UK Collaborative Group for HIV and STI Surveillance. Testing times. HIV and other sexually transmitted infections in the United Kingdom: 2007. London: Health Protection Agency, Centre for Infections, November 2007.

14. Catchpole MA, McGarrigle CA, Rogers PA, et al. Serosurveillance of prevalence of undiagnosed HIV-1 infection in homosexual men with acute sexually transmitted infection. BMJ 2000;321:1319-20.

15. Brown AE, Tomkins SE, Logan LE, et al. Monitoring the effectiveness of HIV and STI prevention initiatives in England, Wales, and Northern Ireland: Where are we now? Sex Transm Infect 2006;82:4-10.

16. Murphy G, Charlett A, Osner N, et al. Reconciling HIV incidence results from two assays employed in the serological testing algorithm for recent HIV seroconversion (STARHS). J Virol Methods 2003;113:79-86.
17. Tatt ID, Barlow KL, Clewley JP, et al. Surveillance of HIV-1 subtypes among heterosexuals in England and Wales, 1997-2000. J Acquir Immune Defic Syndr 2004;36:1092-9.

18. Shafer RW, Rhee SY, Pillay D, et al. HIV-1 protease and reverse transcriptase mutations for drug resistance surveillance. AIDS 2007:21:215-23.

19. Swofford DL. PAUP 4.0: Phylogenetic analysis using parsimony (and other methods). Version 4. Sunderland, MA: Sinaur Associates, 2001.

20. Munro HL, Lowndes CM, Daniels DG, et al. National study of HIV testing in men who have sex with men attending genitourinary clinics in the United Kingdom. Sex Transm Inf 2008; 84:265-70.

21. Hue S, Clewley JP, Cane PA, et al. HIV-1 pol gene variation is sufficient for reconstruction of transmissions in the era of antiretroviral therapy. AIDS 2004;18:719-28.

22. Busch MP, Lee LL, Satten G, et al. Time course of detection of viral and serologic markers pereceding human immunodeficiency virus type 1 seroconversion: implications for screening of blood and tissue donors. Transfusion 1995;35:91-7. 